\title{
ARTICLE
}

\section{Holey two-dimensional transition metal oxide nanosheets for efficient energy storage}

Lele Peng ${ }^{1, \star}$, Pan Xiong ${ }^{1, \star}$, Lu Ma ${ }^{2}$, Yifei Yuan ${ }^{2}$, Yue Zhu ${ }^{1}$ Dahong Chen ${ }^{1}$, Xiangyi Luo ${ }^{2}$, Jun Lu², Khalil Amine ${ }^{2} \&$ Guihua $\mathrm{Yu}^{1}$

Transition metal oxide nanomaterials are promising electrodes for alkali-ion batteries owing to their distinct reaction mechanism, abundant active sites and shortened ion diffusion distance. However, detailed conversion reaction processes in terms of the oxidation state evolution and chemical/mechanical stability of the electrodes are still poorly understood. Herein we explore a general synthetic strategy for versatile synthesis of various holey transition metal oxide nanosheets with adjustable hole sizes that enable greatly enhanced alkali-ion storage properties. We employ in-situ transmission electron microscopy and operando X-ray absorption structures to study the mechanical properties, morphology evolution and oxidation state changes during electrochemical processes. We find that these holey oxide nanosheets exhibit strong mechanical stability inherited from graphene oxide, displaying minimal structural changes during lithiation/delithiation processes. These holey oxide nanosheets represent a promising material platform for in-situ probing the electrochemical processes, and could open up opportunities in many energy storage and conversion systems.

\footnotetext{
${ }^{1}$ Materials Science and Engineering Program and Department of Mechanical Engineering, The University of Texas at Austin, Austin, Texas 78712, USA.

${ }^{2}$ Chemical Sciences and Engineering Division, Argonne National Laboratory, Argonne, Illinois 60439, USA. * These authors contributed equally to this work. Correspondence and requests for materials should be addressed to G.Y. (email: ghyu@austin.utexas.edu) or to J.L. (email: junlu@anl.gov).
} 
T wo-dimensional (2D) nanocrystals offer exciting opportunities for both fundamental studies and many technological applications due to their unique and fascinating properties ${ }^{1-3}$. This has been highlighted over the past decade by 2D graphene and transition metal dichacolgenides, which exhibit exceptional chemical/physical properties that are absent in their bulk counterparts and other dimensional nanostructures ${ }^{4-8}$. Mixed transition metal oxide (MTMO) nanomaterials have been widely studied as attractive candidates for electrocatalysis, photocatalysis, energy storage and conversion technologies, owing to their mixed valence states and rich redox reactions ${ }^{9-13}$. Inspired by the success of $2 \mathrm{D}$ nanomaterials as mentioned above, it is of great interest to fabricate the MTMO materials into 2D nanostructures, which are expected to exhibit greatly improved properties in many energyrelated applications, especially alkali ion storage. However, up to now, MTMOs are mainly studied in the form of $0 \mathrm{D}$ nanoparticles $^{14,15}, 1 \mathrm{D}$ nanotubes or nanowires ${ }^{16,17}$, and 3D nanoclusters or microspheres ${ }^{18}$. In contrast, there are only a few reports studying the $2 \mathrm{D}$ nanostructured MTMOs, especially those with confined thickness ${ }^{19-21}$. MTMOs are intrinsically nonlayered materials, which cannot be mechanically or chemically exfoliated to $2 \mathrm{D}$ nanostructures via the general top-down exfoliation methods ${ }^{22-24}$. Therefore, a general and facile bottom-up strategy for controlled synthesis of 2D MTMO nanostructures is highly needed.

Although 2D nanomaterials generally exhibit improved capacity, rate capability and cycling stability for $\mathrm{Li}$ ion storage due to the abundant active sites for electrochemical reaction and the shortened $\mathrm{Li}$ ion diffusion distance, they still suffer from several disadvantages. $2 \mathrm{D}$ nanomaterials with high surface areas may consume more electrolytes for the formation of SEI, and cause more unwanted side reactions to deteriorate the battery performance ${ }^{2,25,26}$. 2D nanomaterials suffer from decrease of active surfaces for ion transport and storage due to the irreversible restacking of individual $2 \mathrm{D}$ nanosheets ${ }^{27,28}$. Moreover 2D TMO nanomaterials also suffer from severe morphology changes and structural degradation, especially in the first discharge cycle, due to the reduction of the metal cation to $\mathrm{M}(0)$ (refs 29-31). For example, Tarascon et al. reported cobalt oxide $(\mathrm{CoO})$ nanoparticles as a good anode material with high $\mathrm{Li}^{+}$storage capacity and capacity retention. The authors proved that when $\mathrm{CoO}$ nanoparticles were fully reduced by $\mathrm{Li}$, the overall shape of the starting material can be preserved because the disintegrated metallic nanoparticles would be dispersed in a lithia $\left(\mathrm{Li}_{2} \mathrm{O}\right)$ matrix, with the $\mathrm{Li}_{2} \mathrm{O}$ + nanoparticles being surrounded by a solid electrolyte interface (SEI) ${ }^{32}$. Over the past decades, there is a great amount of research on simple and mixed transition metal oxides as lithium ion battery and sodium ion battery anodes ${ }^{33-36}$, but detailed conversion reaction process in terms of the oxidation state changes (chemical stability) and morphology evolution (mechanical stability) are still relatively poorly understood. Meanwhile, the chemical/mechanical stability of these metal oxides and the detailed understanding of their oxidation state changes during electrochemical processes are crucial for further optimization of electrochemical performance.

Here we report a general two-step strategy for controlled synthesis of holey 2D TMO nanosheets with tunable pore sizes using graphene oxides as a sacrificial template (Fig. 1). This approach has been demonstrated to synthesize various $2 \mathrm{D}$ holey TMO nanosheets, including simple oxides such as $\mathrm{Fe}_{2} \mathrm{O}_{3}, \mathrm{Co}_{3} \mathrm{O}_{4}$, $\mathrm{Mn}_{2} \mathrm{O}_{3}$, and mixed oxides such as $\mathrm{ZnMn}_{2} \mathrm{O}_{4}$ (ZMO), $\mathrm{ZnCo}_{2} \mathrm{O}_{4}$ (ZCO), $\mathrm{NiCo}_{2} \mathrm{O}_{4}$ (NCO) and $\mathrm{CoFe}_{2} \mathrm{O}_{4}$ (CFO). As a result, 2D holey TMO nanosheets exhibit much improved rate capability and cycling stability for both lithium and sodium ion storage, due to the increased surface areas and interfaces, and facile interfacial
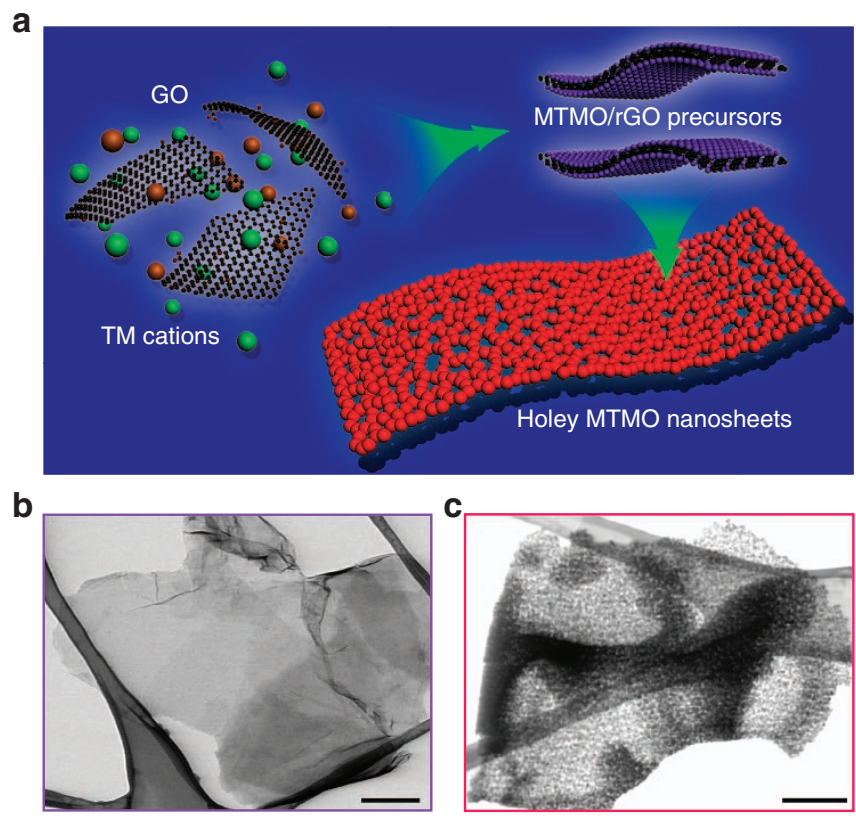

Figure 1 | Schematic illustration of the synthesis process of 2D holey TMO nanosheets. (a) Schematic showing the general strategy to synthesize 2D holey TMO nanosheets. Two transition metal (TM) cations are mixed with graphene oxide (GO) and then anchored on surfaces of reduced graphene oxide $(\mathrm{rGO})$ templates during solution-phase reaction. 2D holey MTMO nanosheets composed of interconnected MTMO nanocrystals are formed after removing $\mathrm{rGO}$ templates during post-calcination. (b) STEM image of ZMO precursor/rGO shows sheets-like morphology. (c) STEM image of 2D holey ZMO nanosheets shows holey nanosheets composed of interconnected ZMO nanocrystals. Scale bars, $200 \mathrm{~nm}(\mathbf{b}, \mathbf{c})$.

transport and shortened diffusion paths. What's more, the operando X-ray diffraction (XRD) and X-ray absorption structures (XAS) are employed to investigate the charge storage mechanism in the conversion reaction involving $2 \mathrm{D}$ holey $\mathrm{ZnMn}_{2} \mathrm{O}_{4}(\mathrm{ZMO})$ nanosheets and to understand the oxidation state changes of $\mathrm{Zn}$ and $\mathrm{Mn}$ elements. We also use in-situ transmission electron microscopy (TEM) to follow the morphology evolution of the $2 \mathrm{D}$ holey $\mathrm{ZMO}$ nanosheets during lithiation/delithiation and pressing process in real time. Operando XRD and XAS results show that holey ZMO nanosheets deliver high capacity due to the formation of $\mathrm{ZnLi}$ alloy as well as the reversible transformation between $\mathrm{Mn}^{2+}$ and $\mathrm{Mn}^{3+}$. In-situ TEM characterizations show that 2D holey ZMO nanosheets composed of chemically interconnected metal oxide nanoparticles inherit the strong mechanical properties from graphene oxide, maintaining the holey morphology and displaying minimal structural changes during the lithiation/ delithiation processes and under press states. The electrochemical results, in combination of the in-situ TEM, operando XRD and XAS studies, show that these $2 \mathrm{D}$ holey nanostructured TMO materials are a promising material platform for both fundamental understanding of the electrode stability during lithiation/ delithiation, and for improving electrochemical performance because of the synergistic effects of the inherently good chemical/mechanical stability and the enhanced charge transport properties.

\section{Results}

Synthesis and characterization of 2D holey MTMO nanosheets. The key concept for general synthesis of $2 \mathrm{D}$ holy TMO nanosheets is illustrated in Fig. 1a. In brief, GO was first 
employed as a template to grow various TMO precursors on its surfaces, followed by post-thermal treatment to transform TMO precursors to $2 \mathrm{D}$ holey TMO nanosheets owing to the synergistic effects of chemical interconnection of TMO nanoparticles and controlled decomposition of GOs. In a typical experiment, TMO precursor/rGO composites were firstly prepared via solutionphase reaction between transition metal ions and GO, which was partially reduced to reduced graphene oxide $(\mathrm{rGO})^{37,38}$. The resulting TMO precursor/rGO were then annealed to induce pyrolysis of rGO templates and formation of crystallized TMO nanoparticles, which interconnected chemically with each other to form the $2 \mathrm{D}$ holey nanosheets. Taking $2 \mathrm{D}$ holey $\mathrm{ZMO}$ nanosheets as an example, GO was firstly dispersed in ethylene glycol solution by ultrasonication. Afterwards, $\mathrm{Zn}\left(\mathrm{CH}_{3} \mathrm{COO}\right)_{2}$ and $\mathrm{Mn}\left(\mathrm{CH}_{3} \mathrm{COO}\right)_{2}$ were added into $\mathrm{GO}$ suspensions. Then stable and homogenous suspensions were obtained by stirring to ensure the complete adsorption of $\mathrm{Zn}^{2+}$ and $\mathrm{Mn}^{2+}$ cations onto the surfaces of GO. After refluxing, the black ZMO precursors/ rGO precipitates were washed and collected by centrifugation. Low magnification scanning transmission electron microscope (STEM) image (Fig. 1b) shows a typical sheet-like morphology of the $\mathrm{ZMO}$ precursors/rGO, indicating that $\mathrm{rGO}$ served as a general template for supporting the ZMO precursors. A close-up STEM image (Supplementary Fig. 1a) indicates that ZMO precursors were well distributed on the rGO sheets. X-ray powder diffraction pattern (Supplementary Fig. 1b) suggests that the as-synthesized ZMO precursors were amorphous. No diffraction peaks of rGO were observed indicating well exfoliation of rGO nanosheets. The STEM (Supplementary Fig. 1c) and corresponding elemental mappings (Supplementary Fig. 1d) show the uniform distributions of $\mathrm{Zn}, \mathrm{Mn}, \mathrm{O}, \mathrm{C}$ elementals, further confirming the $\mathrm{ZMO}$ precursors were uniformly anchored on the surfaces of rGO nanosheets.

Post-calcination of the as-prepared ZMO precursors/rGO induced the transformation of amorphous precursors into crystalline ZMO (Supplementary Fig. 2a) nanosheets without altering their 2D morphology, and simultaneous decomposition of rGO (Supplementary Fig. 2b). The typical STEM images (Fig. 1c and Supplementary Fig. 2c) of the samples after calcination confirm that 2D nanosheet structures had been reserved in the final products. However, the nanosheets had been transformed from a dense structure with smooth surfaces (Fig. 1b and Supplementary Fig. 1a) into a highly holey nanosheet (Fig. 2a,b). The as-synthesized holey nanosheets showed good uniformity in lateral size $(\sim 500 \mathrm{~nm})$ and thickness $(\sim 20 \mathrm{~nm})$ (Supplementary Figs 3 and 4). HRTEM (Fig. 2c) image reveals the 2D holey nanosheets consist of interconnected nanocrystallites of $\sim 8 \mathrm{~nm}$ in size. Moreover, the crossed two fringes in the overlapped domains (Supplementary Fig. 5) reveal that the ZMO nanoparticles indeed are chemically connected with each other. The clear lattice fringes of $\sim 0.25 \mathrm{~nm}$ (Fig. 2c) correspond well to the (211) facet of the spinel ZMO. The diffused concentric rings shown in selected area electron diffraction (SAED) pattern (inset of Fig. 2c) indicate the polycrystalline structure. The diameters of the diffraction rings are indexed to spinel ZMO in agreement with the XRD analysis (Supplementary Fig. 2a). The STEM (Supplementary Fig. 2c) and corresponding elemental mapping (Supplementary Fig. 2d) display the uniform distribution of $\mathrm{Zn}, \mathrm{Mn}$, O elements with rare $\mathrm{C}$ element, which further demonstrates that the $2 \mathrm{D}$ holey nanosheets are composed of the ZMO nanoparticles.

$\mathrm{GO}$ is playing an important role in the formation of $2 \mathrm{D}$ holey ZMO nanosheets. First, GO is a $2 \mathrm{D}$ template with sufficient oxygen-contained groups, which ensures the growth of $\mathrm{ZMO}$ precursors on its surfaces. This is essential for the formation of 2D nanostructure. Second, unlike the conventional templating process where little interactions exist between the precursors and templates, the ZMO precursors were anchored covalently on rGOs through residual functional group such as carboxyl, hydroxyl and epoxy groups (Supplementary Fig. 6) ${ }^{39,40}$. Owing to the intimate interaction between $\mathrm{ZMO}$ and $\mathrm{rGO}$, the $\mathrm{ZMO}$ nanoparticles partially agglomerated and chemically linked with each other to form the holey nanosheets during the thermal treatment, as identified by the HRTEM of ZMO nanoparticles. Third, rGO template is highly flexible, which may contribute to maintaining the structure stability of $2 \mathrm{D}$ holey nanosheets during calcination. Owing to the chemically integration between ZMO precursors and GO, the as-synthesized ZMO nanoparticles inherited the strong mechanical properties from the GO nanosheets. Free ZMO was synthesized via the same method without any GO added as a control experiment. Only an aggregated flower-like structure assembled by spinel ZMO discs was obtained (Supplementary Fig. 7a,b). No holey nanosheet structures were formed (Supplementary Fig. 7c,d).

In many electrochemical energy devices, it is highly desirable to control nanomaterials with tunable pore/hole sizes, since the opening of holes or pores may have a significant effect on their electrochemical performances ${ }^{41,42}$. This is particularly true for 2D nanomaterials as when processed as electrode materials, restacking of nanosheets often leads to substantially reduced active surfaces for electrochemical reactions and suppressed mass transport. In our work, the hole size of 2D holey ZMO nanosheets prepared by such strategy can be adjustable by readily tuning the annealing temperature during post-calcination process. Figure 2b,d,e shows STEM images of $2 \mathrm{D}$ holey ZMO nanosheets prepared by calcination at 400,500 and $600{ }^{\circ} \mathrm{C}$, respectively. The $2 \mathrm{D}$ holey nanosheets structure was well maintained at all temperatures, while the ZMO particles grew bigger and showed more agglomerated as the temperature increases. From Fig. $2 \mathrm{f}$ it is evident that the hole size of $2 \mathrm{D}$ holey ZMO nanosheets increased with the increasing calcination temperature. The hole sizes of $2 \mathrm{D}$ holey $\mathrm{ZMO}$ nanosheets obtained at different temperatures were further tested and verified by the BET characterizations (Supplementary Fig. 8). The BET results gave the average hole sizes of $5 \sim 10,7 \sim 17$ and $\sim 20 \mathrm{~nm}$ for the samples obtained at 400,500 and $600{ }^{\circ} \mathrm{C}$, respectively, which were consistent with the values obtained from the STEM images.

More importantly, such strategy is versatile and can be extended to prepare other 2D holey MTMO nanosheets. Supplementary Fig. 9a,d,g shows scanning electron microscope (SEM) images of $2 \mathrm{D}$ holey $\mathrm{ZnCo}_{2} \mathrm{O}_{4}(\mathrm{ZCO}), \mathrm{NiCo}_{2} \mathrm{O}_{4}(\mathrm{NCO})$ and $\mathrm{CoFe}_{2} \mathrm{O}_{4}$ (CFO) nanosheets prepared by the same approach, respectively. STEM images of $2 \mathrm{D}$ holey $\mathrm{ZnCo}_{2} \mathrm{O}_{4}$ (ZCO), $\mathrm{NiCo}_{2} \mathrm{O}_{4}$ (NCO) and $\mathrm{CoFe}_{2} \mathrm{O}_{4}$ (CFO) nanosheets were shown in Supplementary Fig. 9b,e,h, respectively. In comparison with the 2D ZMO nanosheets shown in Fig. 2a,b, similar 2D holey structures were clearly observed in all these cases. HRTEM (Supplementary Fig. 9c,f,i) images reveal these 2D holey nanosheets all consist of chemically interconnected MTMO nanocrystallites. The lattice fringes (Supplementary Fig. 9c,f,i) and SAED patterns (inset of Supplementary Fig. 9c,f,i) confirm that the 2D ZCO, NCO and CFO nanosheets were composed of spinel ZCO, NCO and CFO, respectively, corresponding well to XRD results (Supplementary Fig. 10). In addition to MTMOs shown above, this strategy can be also applied to the synthesis of simple TMOs, such as $\mathrm{Fe}_{2} \mathrm{O}_{3}, \mathrm{Co}_{3} \mathrm{O}_{4}$, and $\mathrm{Mn}_{2} \mathrm{O}_{3}$. The STEM images displaying the $2 \mathrm{D}$ holey morphology of simple TMOs were shown in Supplementary Fig. 11.

2D holey MTMO nanosheets as anodes for lithium-ion battery. Prompted by their unique morphology, these 2D holey MTMO 

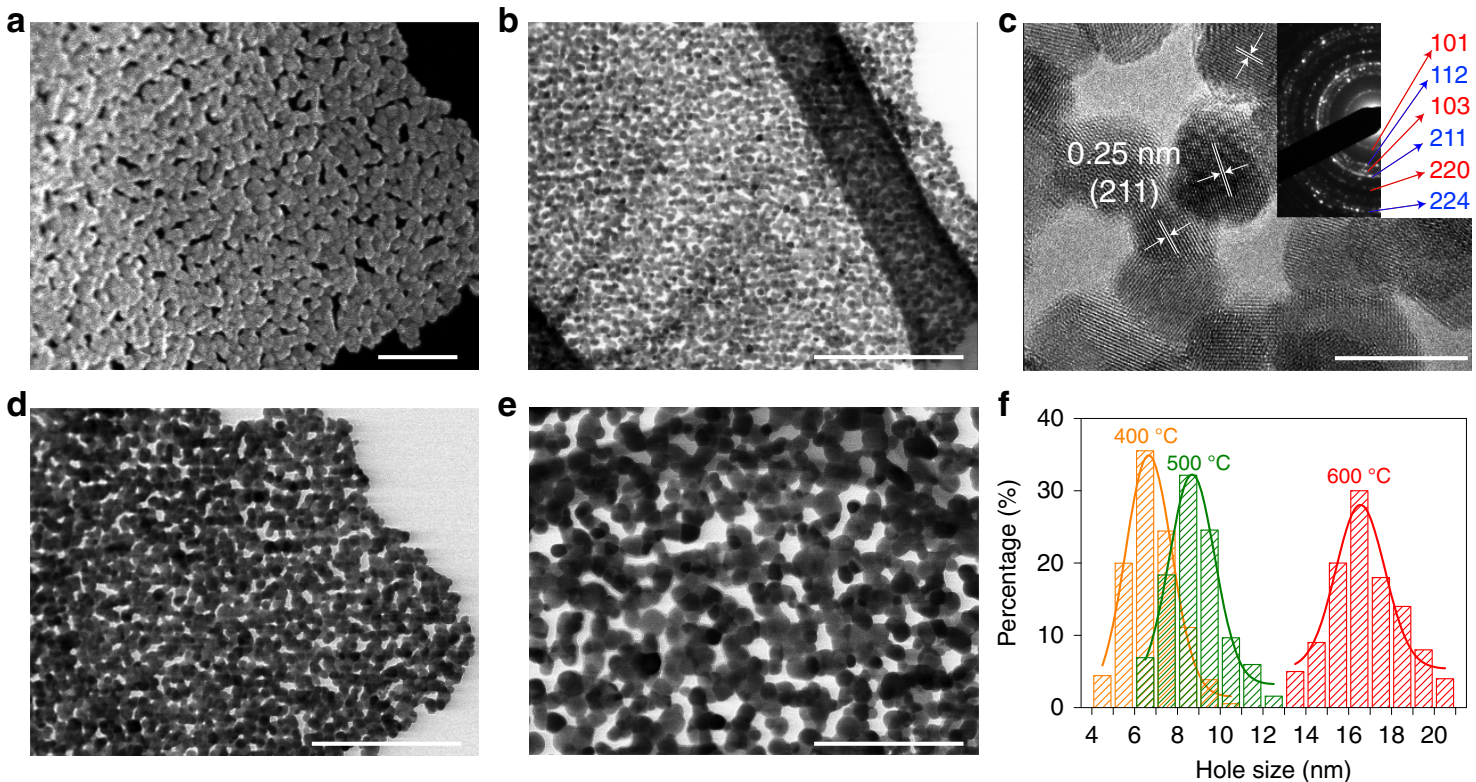

Figure 2 | Electron microscopic images of 2D holey ZMO nanosheets. SEM image (a), STEM image (b) and high-magnification TEM image (c) of 2D holey ZMO nanosheets prepared at post-calcination temperature of $400^{\circ} \mathrm{C}$. Corresponding SAED pattern of $2 \mathrm{D}$ holey ZMO nanosheets (inset of $\mathbf{c}$ ). STEM image of $2 \mathrm{D}$ holey $\mathrm{ZMO}$ nanosheets prepared at different post-calcination temperature: (d) $500^{\circ} \mathrm{C}$ and $(\mathbf{e}) 600^{\circ} \mathrm{C}$. Corresponding hole size distribution (f) obtained by statistics analysis of the STEM images shown in $\mathbf{b}, \mathbf{d}, \mathbf{e}$. Scale bars, $100 \mathrm{~nm}(\mathbf{a}), 200 \mathrm{~nm}(\mathbf{b}, \mathbf{d}, \mathbf{e}), 10 \mathrm{~nm}(\mathbf{c})$.

nanosheets are expected to show promising applications in electrochemical energy storage devices. For example, when used as anodes for lithium-ion batteries, these $2 \mathrm{D}$ holey nanosheets may afford large surface areas and enable facile transport of the electrolyte, resulting in rapid charge transfer due to the shortened diffusion paths ${ }^{18}$. Supplementary Fig. 12a shows the charge and discharge curves of 2D holey ZMO nanosheets for the first two cycles in the range of 0.01 and $3.0 \mathrm{~V}$. The voltage profile of the first $\mathrm{Li}^{+}$charge comprises mainly two regions, a large plateau at $0.5 \mathrm{~V}$ associated with the irreversible reaction between $\mathrm{Li}^{+}$and $\mathrm{ZMO}$, which is followed by a slope till $0.01 \mathrm{~V}$. The $\mathrm{Li}^{+}$discharge curve shows no large plateau but only a slope due to oxidation reactions of $\mathrm{Mn}^{0}$ and $\mathrm{Zn}^{0}$ to $\mathrm{Mn}^{2+}$ and $\mathrm{Zn}^{2}+$. The following charge and discharge slopes reflect the reversible reactions between $\mathrm{Mn}^{0}, \mathrm{Zn}^{0}$ and $\mathrm{Mn}^{2+}, \mathrm{Zn}^{2+}$ and $\mathrm{Zn}-\mathrm{Li}$ alloying/ de-alloying reactions. The initial capacity loss is attributed to the formation of $\mathrm{SEI}^{43}$. After several conditioning cycles, the Coulombic efficiency increased to higher than $98 \%$ (Fig. 3a), indicating good reversibility of the above conversion reactions. After initial two cycles for activation, a stable specific capacity of ca. $500 \mathrm{mAhg}^{-1}$ (the mass of active materials were used to calculate specific capacities) can be observed at a current density of $800 \mathrm{mAg}^{-1}$ for 50 cycles. The control ZMO + SP and control ZMO samples (synthesized without GO template) that cycled at the same conditions (Fig. 3a) only deliver specific capacities of ca. 320 and ca. $100 \mathrm{~mA} \mathrm{~h}^{-1}$, respectively.

The rate capability of the as-prepared $2 \mathrm{D}$ holey $\mathrm{ZMO}$ nanosheets is compared with control $\mathrm{ZMO}+\mathrm{SP}$ and control $\mathrm{ZMO}$ as well (Fig. 3b). For the first few cycles at a low current density of $200 \mathrm{mAg}^{-1}$, the 2D holey ZMO nanosheets show an average specific capacity of ca. $770 \mathrm{~mA} \mathrm{~h}^{-1}$ (Supplementary Fig. 12b). A high capacity of $\sim 430 \mathrm{mAhg}^{-1}$ (Supplementary Fig. 12b) with a $\sim 56 \%$ capacity retention can be achieved at a high current density of $1,200 \mathrm{mAg}^{-1}$. An average specific capacity of $\sim 710 \mathrm{mAhg}^{-1}$ at $200 \mathrm{mAhg}^{-1}$ is retained after 110 cycles of charge and discharge at various current densities. However, for control ZMO $+\mathrm{SP}$ and control ZMO, only $\sim 32 \%$ and $\sim 6 \%$ capacity retention are obtained respectively, as current density increased from 200 to $1,200 \mathrm{~mA} \mathrm{~h}^{-1}$. The superior rate performance is also confirmed in the $2 \mathrm{D}$ holey $\mathrm{ZMO}$ sample prepared at different temperatures (Supplementary Figs 13 and 14). The samples treated at 400 and $500{ }^{\circ} \mathrm{C}$ show the comparable electrochemical performance. While a prominent decrease of rate performance is observed when the calcination temperature increased to $600{ }^{\circ} \mathrm{C}$ (Supplementary Fig. 14). The more aggregated structure and bigger particle size may be attributed to the decreased performance. Furthermore, cycling stability measurement of 2D holey ZMO nanosheets is given in Fig. 3c. After five cycles of electrochemical activation at $100 \mathrm{~mA} \mathrm{~g}^{-1}$, a stable specific capacity of ca. $480 \mathrm{~mA} \mathrm{~h}^{-1}$ can be retained after 1,000 cycles at the current density of $800 \mathrm{mAg}^{-1}$. And the average Coulombic efficiency from the 1st to 1,000 th cycle is $99.8 \%$. The excellent Coulombic efficiency can be attributed to the electrochemical activation process, which facilitates the formation of SEI layers ${ }^{44,45}$. The 2D holey CFO, ZCO and NCO nanosheets can also serve as anodes for lithium storage. The $\mathrm{CV}$ curves of the holey ZCO, holey CFO and holey $\mathrm{NCO}$ nanosheets at scanning rates of $0.1 \mathrm{mV} \mathrm{s}^{-1}$ are showed in Supplementary Fig. 15. Long-term cycling stabilities of these 2D holey nanosheets are shown in Supplementary Fig. 16a-c, respectively. All these $2 \mathrm{D}$ holey nanosheets exhibit high cycling stability revealing that this strategy is a general route for synthesis of $2 \mathrm{D}$ holey nanomaterials with exceptional lithium storage ability. The cycling stability and rate capability of these 2D holey MTMO nanosheets are compared with the reported MTMO-based anodes (Supplementary Table 1). The 2D holey MTMO nanosheets exhibit superior performances. To the best of our knowledge, this is the first time that an MTMO-based anode with such a long cycling stability has been reported.

The high rate capability, excellent cyclic stability and Coulombic efficiency of 2D holey ZMO nanosheets can be ascribed to the unique features of $2 \mathrm{D}$ holey nanostructures with favourable properties. Firstly, the $2 \mathrm{D}$ nanosheets with large surface areas and short diffusion length facilitate effective lithium ion transport. Secondly, the interconnected holes on the surfaces of $2 \mathrm{D}$ nanosheets facilitate the liquid electrolyte diffusion into the 

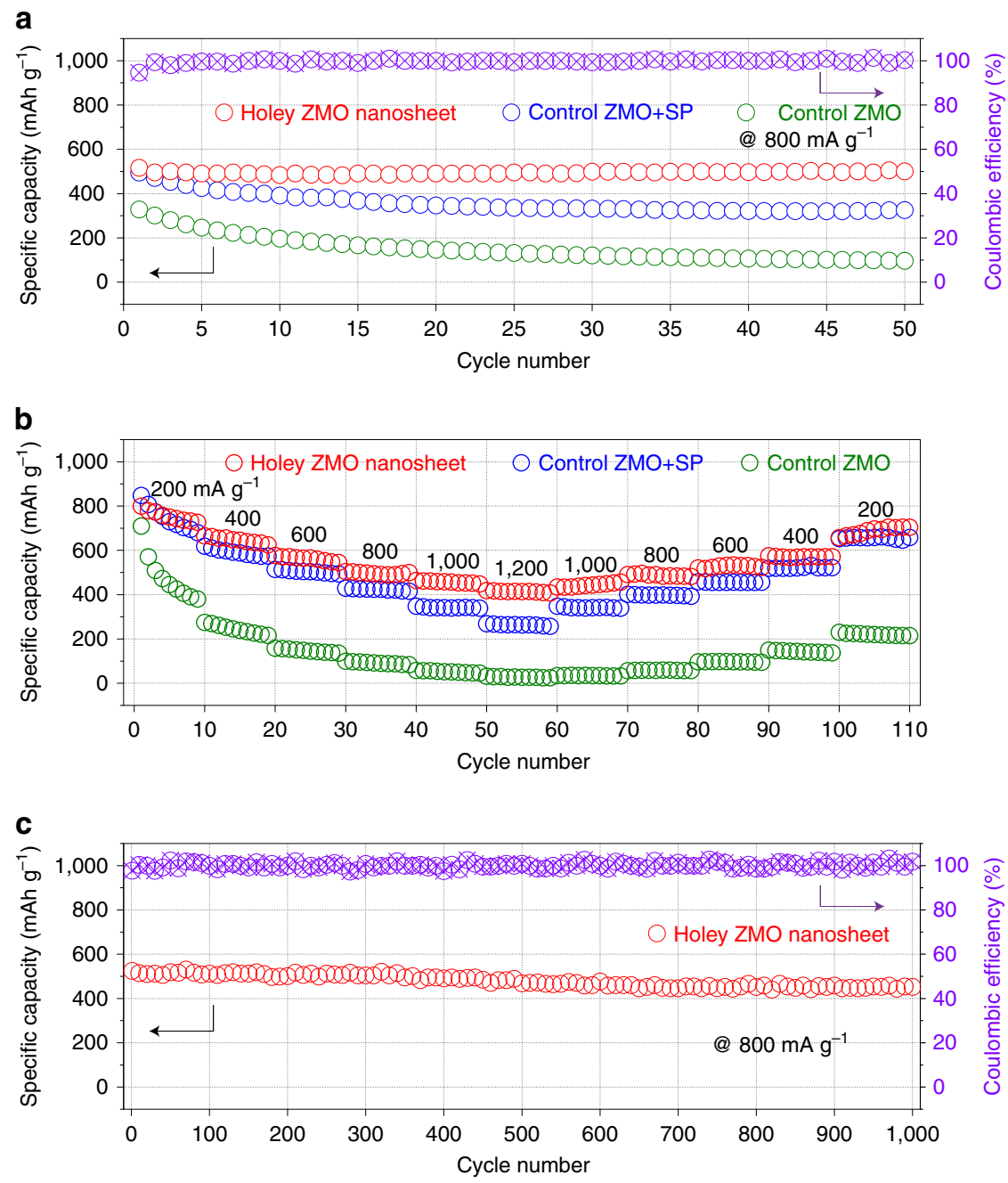

Figure 3 | 2D holey ZMO nanosheets as high-performance anodes for lithium-ion batteries. (a) Cycling performance of 2D holey ZMO nanosheets, control ZMO + SP and control ZMO at current density of $800 \mathrm{mAg}^{-1}$ for 50 cycles. Coulombic efficiency of $2 \mathrm{D}$ holey ZMO nanosheets at current density of $800 \mathrm{mAg}^{-1}$ for 50 cycles. (b) Rate performance of $2 \mathrm{D}$ holey ZMO nanosheets, control ZMO + SP and control ZMO at different current densities from 200 to $1,200 \mathrm{mAg}^{-1}$. (c) Long-term cycling stability and Coulombic efficiency of 2D holey ZMO nanosheet at current density of $800 \mathrm{mAg}^{-1}$ for over 1,000 cycles.

bulk of the electrode materials and greatly reduce the $\mathrm{Li}^{+}$ion diffusion length (Supplementary Fig. 17) ${ }^{42,46}$. Even without adding conductive carbon in the electrodes, electrons could be easily transported continuously along well-interconnected nanocrystals. This is particularly helpful for the battery performance at high rates. Finally, these interconnected holes can help accommodate the volume changes during lithiation/delithiation, thus enhancing the cycling stability and Coulombic efficiency ${ }^{47}$. As shown in Supplementary Fig. 18, 2D holey ZMO nanosheets could still preserve the structure integrity and holey structure after 100 cycles. Such 2D holey nanostructures might endow these materials with many intriguing applications including supercapacitors, catalysis, electrochemical sensors, and so on.

2D holey MTMO nanosheets as anodes for sodium-ion battery. As discussed above, 2D holey MTMO nanosheets possess both 2D nanostructure and tunable porosity, which may contribute to greatly improved electrochemical performance compared to conventional nanosheets electrodes with smooth surfaces. To demonstrate the advantageous features for electrochemical energy storage, 2D holey MTMO nanosheets are further explored as anodes for sodium-ion batteries. The charge/discharge curves of 2D holey NCO nanosheets for the first and second cycles at $100 \mathrm{mAg}^{-1}$ are shown in Fig. 4a. The CV curves of 2D holey NCO nanosheets for first three cycles at $0.1 \mathrm{mV} \mathrm{s}^{-1}$ are shown in Supplementary Fig. 19. In the first discharge cycle, a sloping curve is shown in the potential range of $0.2 \sim 1.2 \mathrm{~V}$ and a flat plateau can be observed at $0.2 \mathrm{~V}$. According to the literature ${ }^{48,49}$, reduction of NCO to metallic $\mathrm{Co}$ and $\mathrm{Ni}$ nanoparticles and formation of $\mathrm{Na}_{2} \mathrm{O}\left(\mathrm{NiCo}_{2} \mathrm{O}_{4}+8 \mathrm{Na}^{+}+8 \mathrm{e}^{-} \rightarrow \mathrm{Ni}+2 \mathrm{Co}+4 \mathrm{Na}_{2} \mathrm{O}\right)$ occurs at the plateau at $0.2 \mathrm{~V}$. The small sloping plateau at $0.7 \mathrm{~V}$ corresponds to the formation of an SEI film. An initial discharge capacity of $\sim 980 \mathrm{mAhg}^{-1}$ and a reversible capacity is $\sim 670 \mathrm{~mA} \mathrm{~h} \mathrm{~g}^{-1}$ with a Coulomb efficiency of $\sim 68.4 \%$ can be achieved. A Coulombic efficiency of around $96 \%$ is reached in the following cycles, indicating that the charge/discharge curves are stabilized gradually. Figure $4 \mathrm{~b}$ and Supplementary Fig. 20 compare the rate performance of the $2 \mathrm{D}$ holey $\mathrm{NCO}$ nanosheets and control NCO nanoplates without porosity (Supplementary Fig. 21) for SIBs. At current densities of 200, $400,800,1,600$ and $3,200 \mathrm{~mA} \mathrm{~g}^{-1}$, the 2D holey NCO nanosheets deliver the capacities of ca. 550, 420, 300, 230 and $170 \mathrm{~mA} \mathrm{~h} \mathrm{~g}^{-1}$, 
a

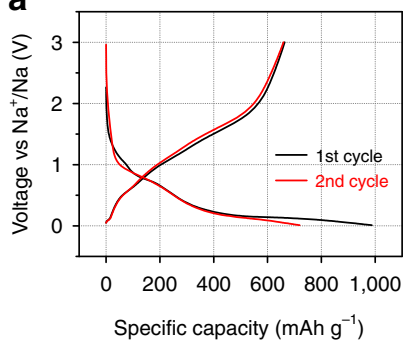

b

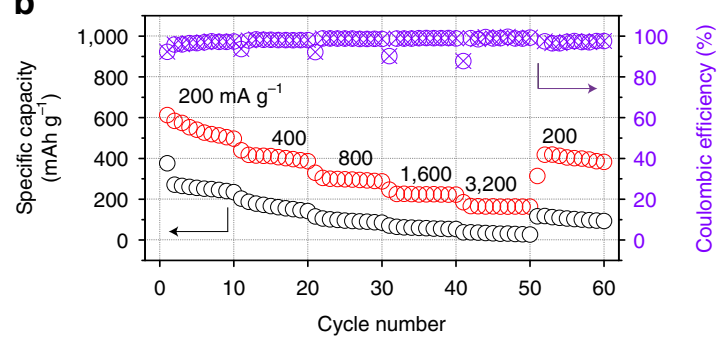

C

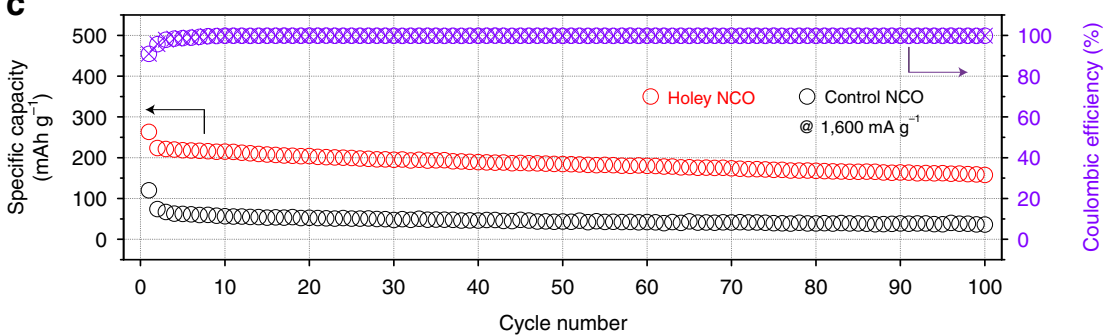

Figure 4 | 2D holey NCO nanosheets as anodes for sodium-ion batteries. (a) Charge/discharge profiles of 2D holey NCO nanosheets for the first and second cycles at a current density of $100 \mathrm{mAg}^{-1}$. (b) Rate performances of 2D holey NCO nanosheets (red circle) and control NCO nanosheets (black circle) from 200 to 3,200 $\mathrm{mAg}^{-1}$. (c) Long-term cycling stability and Coulombic efficiency of 2D holey NCO nanosheet and control NCO nanosheet at current density of $1,600 \mathrm{mAg}^{-1}$ for 100 cycles.

respectively. The holey naosheets electrodes still deliver a reversible capacity of $420 \mathrm{~mA} \mathrm{~h} \mathrm{~g}^{-1}$ when the current density is reverted gradually to $200 \mathrm{mAg}^{-1}$. While the control NCO nanosheets without porosity only deliver a low capacity of $\sim 60 \mathrm{~mA} \mathrm{~h}^{-1}$ at the current density of $1,600 \mathrm{mAg}^{-1}$, and can be hardly charged/discharged at higher current density. Figure $4 \mathrm{c}$ shows the long-term cycling stability and Coulombic efficiency of 2D holey $\mathrm{NCO}$ nanosheets and control $\mathrm{NCO}$ nanosheets at current density of $1,600 \mathrm{~mA} \mathrm{~g}^{-1}$ for 100 cycles. A stable specific capacity of ca. $160 \mathrm{~mA} \mathrm{~h}^{-1}$ was observed after 100 cycles for the 2D holey NCO nanosheet anodes. For comparison, the control anodes, NCO nanosheet without porous structure, showed the specific capacities of ca. $36 \mathrm{~mA} \mathrm{~h}^{-1}$ after 100 cycles. These high rate capability and excellent cyclic stability can be attributed to the unique $2 \mathrm{D}$ holey structure of the NCO nanosheets. The unique 2D holey structure will enable facile diffusion of liquid electrolyte into the bulk of the electrode materials and greatly reduce the $\mathrm{Li}^{+}$ion diffusion length, thus improving the rate capability. Also, these interconnected holes can also maintain the holey framework of the nanosheets and accommodate volume change during charging/discharging, thus improving the cycling stability and Coulombic efficiency.

In-situ TEM studies of 2D holey MTMO nanosheet anodes. The high storage capacity for $\mathrm{Li} / \mathrm{Na}$, high rate capability and cycling stability of $2 \mathrm{D}$ holey nanosheet samples can be ascribed to the $2 \mathrm{D}$ interconnected porous framework that provides large active surface areas, bicontinuous $\mathrm{Li}^{+} / \mathrm{Na}^{+}$and electron pathways and excellent structural stability inherited from the graphene oxide. To further understand the structural stability of 2D holey nanosheets, structural evolution during the lithiation process is carried out by in-situ TEM imaging on ZMO nanosheet, as a model material. The first-cycle lithiation process of $2 \mathrm{D}$ holey ZMO nanosheets is examined, as shown in Fig. 5. As discussed before, $\mathrm{ZMO}$ is fully reduced by $\mathrm{Li}$ into $\mathrm{Zn}$ and $\mathrm{Mn}$ metallic nanoparticles which are dispersed in a lithia $\left(\mathrm{Li}_{2} \mathrm{O}\right)$ matrix, with the $\mathrm{Li}_{2} \mathrm{O}+$ nanoparticles being surrounded by an $\mathrm{SEI}^{33}$. These results can be further identified by SAED of the fresh and lithiated samples, as shown in Supplementary Fig. 22. The SAED of fresh samples can be indexed to the spinel ZMO structures in agreement with the XRD analysis. This result is different from that of the lithiated samples, which can be indexed to the $\mathrm{Zn}$ and Mn metallic phases (Supplementary Fig. 22b). It should be noted that the in-situ TEM characterizations during the first lithiation process proved the structural uniqueness and inherently strong mechanical stability of the 2D holey ZMO nanosheets. During the first lithiation (Fig. 5a-f), 2D holey ZMO nanosheets maintain the holey morphology in different lithiation stages/times, for example 3, 9, 12 and $15 \mathrm{~min}$. After full lithiation, the overall shape of the starting material can be preserved in 2D geometry, and the overall morphology remains as holey/porous structures because the disintegrated metallic nanoparticles would be dispersed in a lithia $\left(\mathrm{Li}_{2} \mathrm{O}\right)$ matrix. The lithiated $\mathrm{ZMO}$ nanosheets can remain its overall morphology and porous nature when the ZMO nanosheet is under press during in-situ stress tests, as shown in Fig. 5g-i. These results are consistent with the aforementioned improved electrochemical characteristics for $\mathrm{Li} / \mathrm{Na}$ ion storage. The $2 \mathrm{D}$ holey MTMO nanosheets can maintain the holey framework and accommodate volume change during charging/discharging, thus improving the cycling stability and Coulombic efficiency.

Operando XAS studies on 2D holey MTMO nanosheets. As the spinel structure of MTMO is not recovered after the first cycle, operando XAS was further applied as a powerful method to provide detailed information about the oxidation states and the local environment changes of atoms on a short-range scale. The XAS scans of the Zn K-edges and Mn K-edge and their corresponding first derivative curves during the charge/discharge processes are shown in Fig. 6. The edge position of the XAS spectra is related to the oxidation state. Initially, the edge energy of the $\mathrm{Zn} \mathrm{K}$-edge was $9,664 \mathrm{eV}$, which is a little higher than that of $\mathrm{Zn}^{2+}$, indicating that the oxidation state of $\mathrm{Zn}$ is a little higher than $2+$ (peak $\mathrm{A}$ in the first derivative plot is at a higher energy than $\mathrm{ZnO}$ standard) (Fig. 6a,b). This is probably due to the formation of the intermediate $\mathrm{NaCl}$-type structure in which the $\mathrm{Zn}^{2+}$ has tetrahedral coordination ${ }^{50,51}$. During formation of the intermediate $\mathrm{NaCl}$-type structure, the intensity of the $1 s \rightarrow 4 p$ transition increases for the $\mathrm{Zn}$ K-edge. For the discharge process, the intensity of this spectral feature progressively decreases, which can be explained by the loss of $p$ character of the $1 s \rightarrow 4 p$ 
a

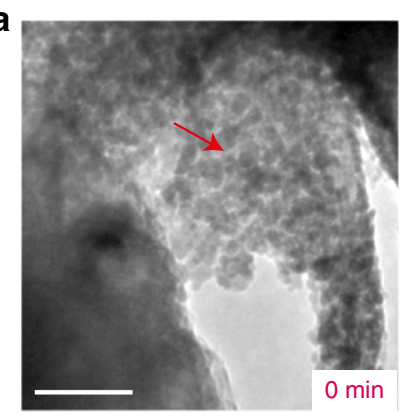

d

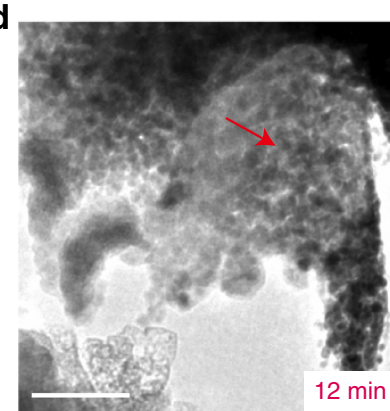

g

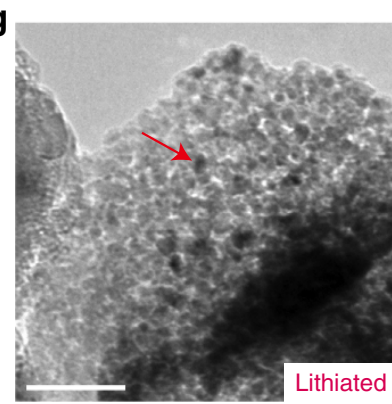

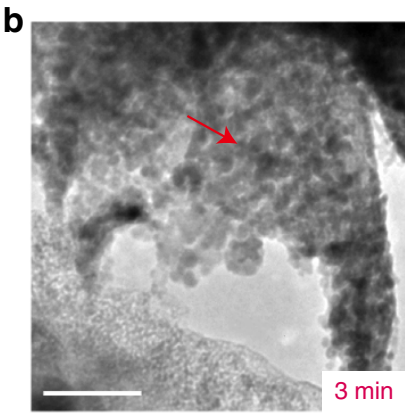
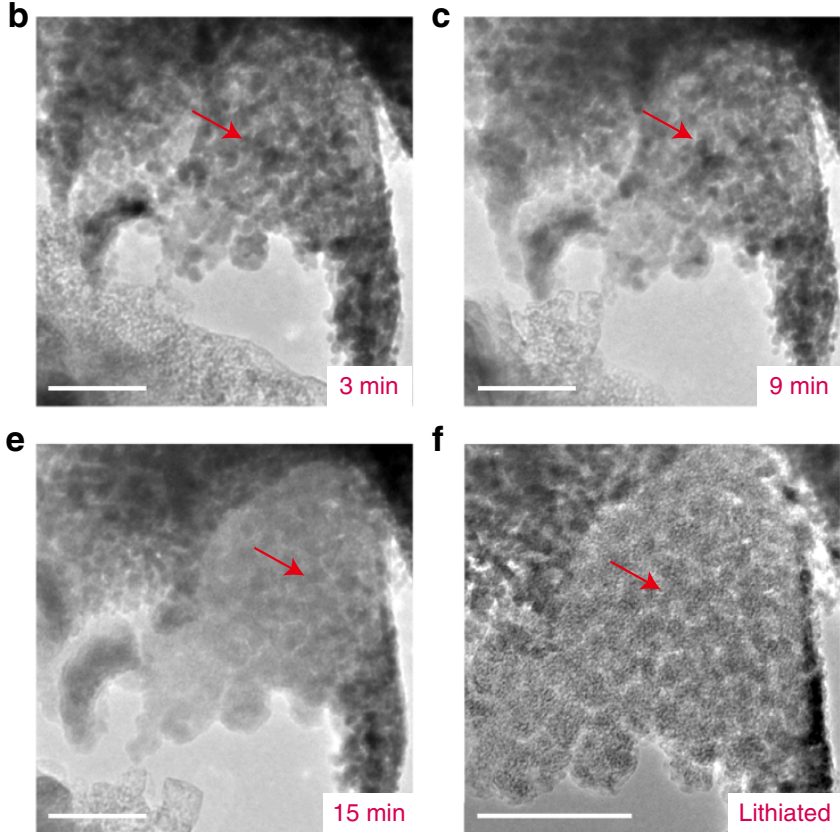

h

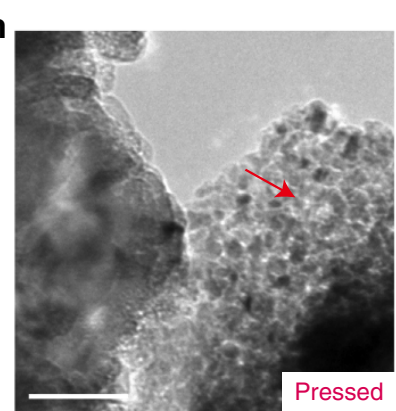

i

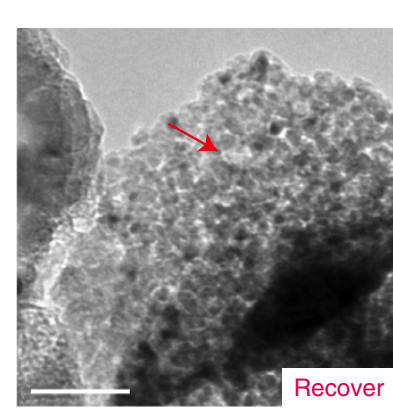

Figure 5 | In-situ TEM studies of 2D holey ZMO nanosheets as anodes. (a-f) TEM images of the 2D holey nanosheets at different lithiation stages/times. (g-i) TEM images of the 2D holey nanosheets under press states. Scale bars, $100 \mathrm{~nm}$. Red arrow points out the holey structures of the 2D ZMO nanosheets.

a

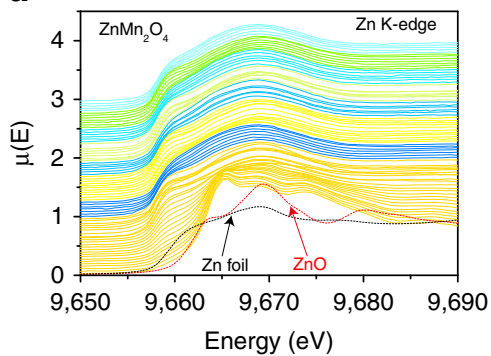

d

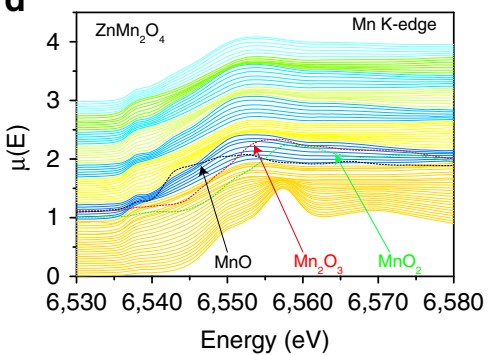

b

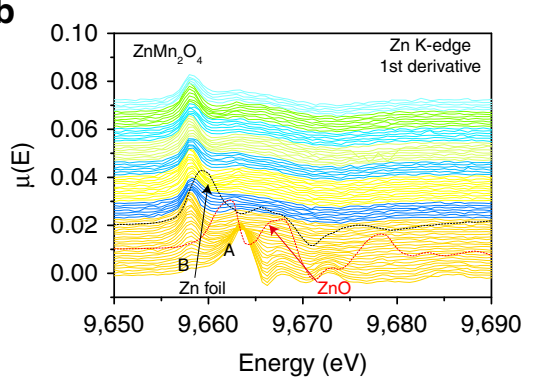

e

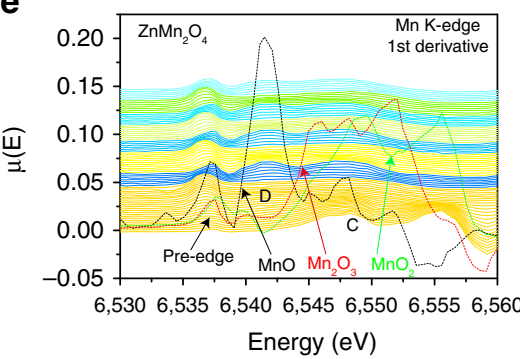

C

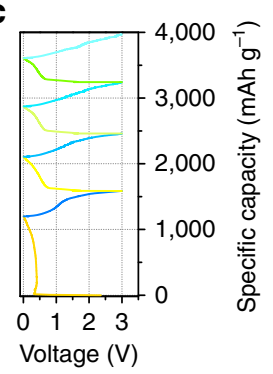

f

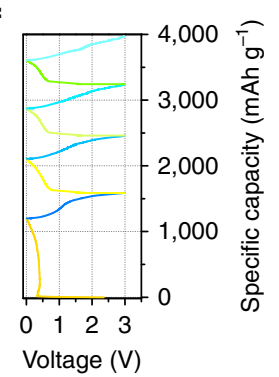

Figure 6 | Operando XAS studies on 2D holey MTMO nanosheet. (a,b) Scan at the Zn K-edge and the corresponding first derivative curves during the charge/discharge process. (c) The charge/discharge curves of the $2 \mathrm{D}$ holey ZMO nanosheets at $50 \mathrm{mAg}^{-1}$. (d,e) Scan at the Mn K-edge and the corresponding first derivative curves during the charge/discharge process. (f) The charge/discharge curves of the 2D holey ZMO nanosheets at $50 \mathrm{mAg}^{-1}$. 
transition as a consequence of the formation of metallic $\mathrm{Zn}$ nanoparticles, which occupies the $4 p$ orbital. Metallic $\mathrm{Zn}$ nanoparticles further form the $\mathrm{ZnLi}$ alloy with $\mathrm{Li}$ after further discharge (Fig. 6c). This fact is identified by the peak position, which showed lower energy than $\mathrm{Zn}$ foil, as shown in first derivative curves. The oxidation state of $\mathrm{Mn}$ before the first discharge cycle is close to $3+$ (peak $\mathrm{C}$ in the first derivative plot at similar position as $\mathrm{Mn}$ in $\mathrm{Mn}_{2} \mathrm{O}_{3}$ ). As the $\mathrm{Mn} \mathrm{K}$-edge and its first derivative curves shown in Fig. 6d,e, the Mn K-edge shows a continuous shift to lower edge energy, that is, the electrons are transferred to $\mathrm{Mn}^{3}+$ during the first discharge cycle. During the first discharge, the intensity of the pre-edge peak increased and the peak $C$ became weak. The pre-edge peak is associated with transition to empty $\mathrm{Mn} 3 d$ states. The single pre-edge peak indicates that the $\mathrm{Mn}$ is still $3+$, but due to the change of the spinel phase, the $1 s \rightarrow 3 d$ transition increases. During the charge process, pre-edge structure changed to double-peaked structure, which indicated the transition of $\mathrm{Mn}^{3+}$ to $\mathrm{Mn}^{2}$. These results correspond well with the Operando XRD results shown in Supplementary Fig. 23. After the first discharge cycle, the oxidation state of $\mathrm{Zn}$ slightly increases during charge and decreases during discharge process, indicating the cycling process between $\mathrm{ZnLi}$ alloy and metallic $\mathrm{Zn}$ nanoparticles. Simultaneously, the oxidation state of $\mathrm{Mn}$ changes from +3 to +2 in the charge process and goes back to +3 at the end of the discharge process, as identified by the reversible shifting of the pre-edge peak positions. The operando XAS studies prove the good chemical stability of the 2D holey ZMO nanosheets during charge/discharge process, showing no unwanted impurities and minimal side reactions occurred in the charge/discharge process.

\section{Discussion}

In this work, a general synthetic strategy is developed by employing graphene oxide as a sacrificial template to prepare various $2 \mathrm{D}$ holey $\mathrm{TMO}$ nanosheets, including mixed metal oxides $\mathrm{ZnMn}_{2} \mathrm{O}_{4}, \mathrm{ZnCo}_{2} \mathrm{O}_{4}, \mathrm{NiCo}_{2} \mathrm{O}_{4}, \mathrm{CoFe}_{2} \mathrm{O}_{4}$, and simple metal oxides, such as $\mathrm{Fe}_{2} \mathrm{O}_{3}, \mathrm{Co}_{3} \mathrm{O}_{4}$, and $\mathrm{Mn}_{2} \mathrm{O}_{3}$. As potential anode materials for both lithium and sodium ion storage, the as-obtained 2D holey MTMO nanosheets exhibit superior electrochemical properties including high reversible capacity, excellent rate capability and cycling stability. This work combined in-situ TEM, XRD, with in-situ XAS measurements, and revealed for the first time the mechanical properties and morphology evolution of the 2D holey MTMO nanosheets during electrochemical processes. Operando XRD and XAS results show that holey ZMO nanosheets deliver high capacity due to the formation of $\mathrm{ZnLi}$ alloy as well as the reversible transformation between $\mathrm{Mn}^{2+}$ and $\mathrm{Mn}^{3+}$. In-situ TEM characterizations show that these $2 \mathrm{D}$ holey ZMO nanosheets inherit the strong mechanical properties from graphene oxides, maintaining the holey morphology and displaying minimal structural changes during the lithiation/delithiation processes and even under press states. Owing to potential merits of 2D nanostructures, tunable porosity and inherently strong mechanical stability, these 2D holey nanosheets could be promising candidates for emerging applications in energy storage and conversion systems, and electrocatalysis. The proposed general strategy would open up a promising avenue for designing $2 \mathrm{D}$ nanostructures for versatile materials, especially those with intrinsically non-layered structures, for broad applications in nanoscience and nanotechnology.

\section{Methods}

Synthesis of graphene oxide (GO). GO was prepared from purified natural graphite by a modified Hummers' method. Simply, $10 \mathrm{~g}$ of graphite powder was first added to $15 \mathrm{ml}$ of concentrated $\mathrm{H}_{2} \mathrm{SO}_{4}$. Five grams of $\mathrm{K}_{2} \mathrm{~S}_{2} \mathrm{O}_{8}$ and $5 \mathrm{~g}$ of $\mathrm{P}_{2} \mathrm{O}_{5}$ were then added slowly. The as-obtained mixed solution was heat-treated at $80^{\circ} \mathrm{C}$ for $6 \mathrm{~h}$. After cooling to room temperature, the resultant was carefully diluted with distilled water, filtered and washed on the filter until the rinse water $\mathrm{pH}$ became neutral. The product was dried in air at ambient temperature overnight. Then the preoxidized graphite was added to $230 \mathrm{ml}$ of concentrated $\mathrm{H}_{2} \mathrm{SO}_{4}$ cooled in an ice-water bath. Thirty grams of $\mathrm{KMnO}_{4}$ was added very slowly into the mixture with stirring and cooling. All the operations were carried out very slowly in a fume hood. The as-obtained mixture was then magnetically stirred at $35^{\circ} \mathrm{C}$ for $30 \mathrm{~min}$ followed by slowly adding $460 \mathrm{ml}$ of distilled water. The temperature of system increased to $98^{\circ} \mathrm{C}$ during this process and was kept for another $15 \mathrm{~min}$. After adding $1.4 \mathrm{l}$ of distilled water followed by $10 \mathrm{ml}$ of $30 \% \mathrm{H}_{2} \mathrm{O}_{2}$ solution, solid products were formed and the reaction was terminated. The solid product was separated by centrifugation, washed repeatedly with $5 \% \mathrm{HCl}$ solution, and then dialysed for a week.

Synthesis of 2D holey MTMO nanosheets. The 2D holey MTMO nanosheets were prepared via a facile two-step approach. In a typical synthesis of $2 \mathrm{D}$ holey ZMO nanosheets, GO suspension was first formed by dispersing $30 \mathrm{mg}$ GO power in $75 \mathrm{ml}$ of ethylene glycol (EG) under sonication. Metal precursors were formed by dissolving $0.5 \mathrm{mmol}$ of zinc acetates $\left(\mathrm{Zn}\left(\mathrm{CH}_{3} \mathrm{COO}\right)_{2} \cdot 2 \mathrm{H}_{2} \mathrm{O}\right)$ and $1.0 \mathrm{mmol}$ of manganese acetates $\left(\mathrm{Mn}\left(\mathrm{CH}_{3} \mathrm{COO}\right)_{2} \cdot 4 \mathrm{H}_{2} \mathrm{O}\right)$ in $25 \mathrm{ml}$ of EG. The above two systems were then mixed together, and stirred at ambient temperature for at least $60 \mathrm{~min}$ to obtain a homogenous suspension. The obtained suspension was heated at $170{ }^{\circ} \mathrm{C}$ for $2 \mathrm{~h}$ in a reflux synthesis system. The mixture was then allowed to naturally cool down, and the products were obtained by centrifuging and washing with ethanol for several times. The product (donated as ZMO precursors/GO) was then dried in vacuum at $80^{\circ} \mathrm{C}$ overnight. In the second step of synthesis, the $2 \mathrm{D}$ holey ZMO nanosheets can be obtained through calcination of the as-made ZMO precursors/GO at $400^{\circ} \mathrm{C}$ in air for $120 \mathrm{~min}$ with ramp rate of $0.5^{\circ} \mathrm{C} \mathrm{min}-1$. To prepare control ZMO sample, same amount of the chemicals and EG solvent were used for the reaction without any GO added as the typical synthesis mentioned above. The rGO was prepared via the first step of the synthesis without $\mathrm{Zn}\left(\mathrm{CH}_{3} \mathrm{COO}\right)_{2} \cdot 2 \mathrm{H}_{2} \mathrm{O}$ and $\mathrm{Mn}\left(\mathrm{CH}_{3} \mathrm{COO}\right)_{2} \cdot 4 \mathrm{H}_{2} \mathrm{O}$. To evaluate the influence of calcination temperature, the as-made $\mathrm{ZMO}$ precursors/GO was annealed at 400 , 500 and $600^{\circ} \mathrm{C}$ in air for $120 \mathrm{~min}$ with same heating rate $\left(0.5^{\circ} \mathrm{C} \mathrm{min}{ }^{-1}\right)$, donated as 2D holey ZMO-400, 2D holey ZMO-500, 2D holey ZMO-600, respectively. $2 \mathrm{D}$ holey $\mathrm{ZCO}$ nanosheets were prepared with $\mathrm{Zn}\left(\mathrm{CH}_{3} \mathrm{COO}\right)_{2} \cdot 2 \mathrm{H}_{2} \mathrm{O}$ and $\mathrm{Co}\left(\mathrm{CH}_{3} \mathrm{COO}\right)_{2} \cdot 2 \mathrm{H}_{2} \mathrm{O}$ in the presence of $\mathrm{GO}$ as the same method mentioned above. $2 \mathrm{D}$ holey NCO nanosheets were prepared by replacing $\mathrm{Zn}\left(\mathrm{CH}_{3} \mathrm{COO}\right)_{2} \cdot 2 \mathrm{H}_{2} \mathrm{O}$ and $\mathrm{Mn}\left(\mathrm{CH}_{3} \mathrm{COO}\right)_{2} \cdot 4 \mathrm{H}_{2} \mathrm{O}$ with $\mathrm{Ni}\left(\mathrm{CH}_{3} \mathrm{COO}\right)_{2} \cdot 4 \mathrm{H}_{2} \mathrm{O}$ and $\mathrm{Co}\left(\mathrm{CH}_{3} \mathrm{COO}\right)_{2} \cdot 4 \mathrm{H}_{2} \mathrm{O}$ as the same method mentioned above. $2 \mathrm{D}$ holey $\mathrm{CFO}$ nanosheets were prepared by replacing $\mathrm{Zn}\left(\mathrm{CH}_{3} \mathrm{COO}\right)_{2} \cdot 2 \mathrm{H}_{2} \mathrm{O}$ and $\mathrm{Mn}\left(\mathrm{CH}_{3} \mathrm{COO}\right)_{2} \cdot 4 \mathrm{H}_{2} \mathrm{O}$ with $\mathrm{Co}\left(\mathrm{NO}_{3}\right)_{2} \cdot 6 \mathrm{H}_{2} \mathrm{O}$ and $\mathrm{Fe}\left(\mathrm{NO}_{3}\right)_{3} \cdot 9 \mathrm{H}_{2} \mathrm{O}$ as the same method mentioned above.

Characterization. Powder XRD patterns were collected on a Philips Vertical Scanning difractometer to identify the phase of the as-synthesized samples. Scanning electron microscope, STEM (Hitachi S5500) and TEM (JEOL 2010F) were used to characterize the morphology of the samples. The TG analysis was tested by a TGA/SDTA851e thermogravimetric analyser under an air atmosphere from 25 to $850^{\circ} \mathrm{C}$ at a heating rate of $10^{\circ} \mathrm{C} \mathrm{min}^{-1}$. In-situ TEM (JEOL 3,010) was carried out using a Nanofactory holder capable of biasing. In the beginning, $2 \mathrm{D}$ holey $\mathrm{ZnMn}_{2} \mathrm{O}_{4}$ nanosheets were loaded to one gold tip by conductive glue, and metallic Li was attached to the other Tungsten tip. During the sample transfer into the TEM chamber, Li metal was exposed to air for about $30 \mathrm{~s}$ and was oxidized to $\mathrm{Li}_{2} \mathrm{O}$ on the surface, which acts as the solid electrolyte. After the samples were loaded in the TEM, a $+3 \mathrm{~V}$ bias (on the $\mathrm{Li} / \mathrm{Li}_{2} \mathrm{O}$ side) was applied to initiate the lithiation process. The mechanical press/release was carried out after $\mathrm{ZnMn}_{2} \mathrm{O}_{4}$ nanosheets is fully lithiated and the bias is off.

Electrochemical measurements. The working electrodes were prepared by mixing active materials (2D holey ZMO nanosheets) and polyvinylidene difluoride at a weight ratio of 90:10, in $N$-methyl-2-pyrrolidinone. Then the slurries were coated onto a copper foil. The as-prepared electrodes were dried under vacuum at $110^{\circ} \mathrm{C}$ for $10 \mathrm{~h}$. The loading of active materials was $\sim 1.0 \mathrm{mg} \mathrm{cm}^{-2}$. After being sealed, the electrodes were assembled into coin cells (CR2032) in an argon-filled glovebox using Celgard 2320 as separator, $1 \mathrm{moll}^{-1} \mathrm{LiPF}_{6}$ in ethylenecarbonate and diethylenecarbonate $(1: 1, \mathrm{v} / \mathrm{v})$ as the electrolyte and $\mathrm{Li}$ metal as the counter electrode. The assembled coin cells were tested on an Arbin battery test system with a voltage range of $0.01 \sim 3.0 \mathrm{~V}$. For comparison, the control electrode, named as Control ZMO, is made of free ZMO nanoplates without holes. Another control electrode, named as $\mathrm{ZMO}+\mathrm{SP}$, is made of free $\mathrm{ZMO}$ nanoplates physically mixed with Super P carbon in the weight ratio of 75:25. The 2D holey NCO nanosheets based electrode for sodium ion batteries were prepared by mixing active materials (2D holey NCO nanosheets), superP, sodium carboxymethyl cellulose (CMC) at a weight ratio of 80:10:10, in water. The electrolyte used for sodium ion battery tests was $1 \mathrm{M} \mathrm{NaClO}_{4}$ dissolved in propylene carbonate with $2 \%$ fluoroethylene carbonate additive.

Data availability. The data that support the findings of this study are available from the corresponding authors on request. 


\section{References}

1. Huang, X., Tan, C., Yin, Z. \& Zhang, H. 25th anniversary article: hybrid nanostructures based on two-dimensional nanomaterials. Adv. Mater 26, 2185-2204 (2014)

2. Bonaccorso, F. et al. Graphene, related two-dimensional crystals, and hybrid systems for energy conversion and storage. Science 347, 41 (2015).

3. Anasori, B. et al. Two-dimensional, ordered, double transition metals carbides (MXenes). ACS Nano 9, 9507-9516 (2015).

4. Zhu, Y. et al. Graphene and graphene oxide: synthesis, properties, and applications. Adv. Mater. 22, 3906-3924 (2010).

5. Novoselov, K. S. et al. Two-dimensional atomic crystals. Proc. Natl Acad. Sci. USA 102, 10451-10453 (2005).

6. Chhowalla, M. et al. The chemistry of two-dimensional layered transition metal dichalcogenide nanosheets. Nat. Chem. 5, 263-275 (2013).

7. Geim, A. K. \& Novoselov, K. S. The rise of graphene. Nat. Mater. 6, 183-191 (2007).

8. Wang, Q. H., Kalantar-Zadeh, K., Kis, A., Coleman, J. N. \& Strano, M. S. Electronics and optoelectronics of two-dimensional transition metal dichalcogenides. Nat. Nanotechnol. 7, 699-712 (2012).

9. Yuan, C., Wu, H. B., Xie, Y. \& Lou, X. W. Mixed transition-metal oxides: design, synthesis, and energy-related applications. Angew. Chem. Int. Ed. 53, 1488-1504 (2014)

10. Liang, Y. et al. Covalent hybrid of spinel manganese-cobalt oxide and graphene as advanced oxygen reduction electrocatalysts. J. Am. Chem. Soc. 134, 3517-3523 (2012).

11. Cheng, F. et al. Rapid room-temperature synthesis of nanocrystalline spinels as oxygen reduction and evolution electrocatalysts. Nat. Chem. 3, 79-84 (2011)

12. Xiong, P. et al. Chemically integrated two-dimensional hybrid zinc manganate/ graphene nanosheets with enhanced lithium storage capability. ACS Nano 8, 8610-8616 (2014).

13. Xiong, P. et al. Two-dimensional nanosheets based Li-ion full batteries with high rate capability and flexibility. Nano Energy 12, 816-823 (2015).

14. Zeng, H., Rice, P. M., Wang, S. X. \& Sun, S. Shape-controlled synthesis and shape-induced texture of $\mathrm{MnFe}_{2} \mathrm{O}_{4}$ nanoparticles. J. Am. Chem. Soc. 126, 11458-11459 (2004).

15. Song, Q. \& Zhang, Z. J. Controlled synthesis and magnetic properties of bimagnetic spinel ferrite $\mathrm{CoFe}_{2} \mathrm{O}_{4}$ and $\mathrm{MnFe}_{2} \mathrm{O}_{4}$ nanocrystals with core-shell architecture. J. Am. Chem. Soc. 134, 10182-10190 (2012).

16. Liu, B. et al. Hierarchical three-dimensional $\mathrm{ZnCo}_{2} \mathrm{O}_{4}$ nanowire arrays/carbon cloth anodes for a novel class of high-performance flexible lithium-ion batteries. Nano Lett. 12, 3005-3011 (2012).

17. Huang, L. et al. Nickel-cobalt hydroxide nanosheets coated on $\mathrm{NiCo}_{2} \mathrm{O}_{4}$ nanowires grown on carbon fiber paper for high-performance pseudocapacitors. Nano Lett. 13, 3135-3139 (2013).

18. $\mathrm{Hu}$, L. et al. $\mathrm{CoMn}_{2} \mathrm{O}_{4}$ spinel hierarchical microspheres assembled with porous nanosheets as stable anodes for lithium-ion batteries. Sci. Rep. 2, 986 (2012).

19. Zhu, Y., Cao, C., Zhang, J. \& Xu, X. Two-dimensional ultrathin $\mathrm{ZnCo}_{2} \mathrm{O}_{4}$ nanosheets: general formation and lithium storage application. J. Mater. Chem. A 3, 9556-9564 (2015).

20. Zhu, Y. \& Cao, C. A simple synthesis of two-dimensional ultrathin nickel cobaltite nanosheets for electrochemical lithium storage. Electrochim. Acta 176, 141-148 (2015)

21. Sun, Z. et al. Generalized self-assembly of scalable two-dimensional transition metal oxide nanosheets. Nat. Commun. 5, 4813 (2014).

22. Zhou, K.-G., Mao, N.-N., Wang, H.-X., Peng, Y. \& Zhang, H.-L. A mixed-solvent strategy for efficient exfoliation of inorganic graphene analogues. Angew. Chem. Int. Ed. 50, 10839-10842 (2011).

23. Nicolosi, V., Chhowalla, M., Kanatzidis, M. G., Strano, M. S. \& Coleman, J. N. Liquid exfoliation of layered materials. Science 340, 6139 (2013).

24. Coleman, J. N. et al. Two-dimensional nanosheets produced by liquid exfoliation of layered materials. Science 331, 568-571 (2011).

25. Liu, J. \& Liu, X.-W. Two-dimensional nanoarchitectures for lithium storage. Adv. Mater. 24, 4097-4111 (2012).

26. Peng, L., Zhu, Y., Chen, D., Ruoff, R. S. \& Yu, G. Two-dimensional materials for beyond-lithium-ion batteries. Adv. Energy Mater. 6, 1600025 (2016).

27. Acerce, M., Voiry, D. \& Chhowalla, M. Metallic 1 T phase $\mathrm{MoS}_{2}$ nanosheets as supercapacitor electrode materials. Nat. Nanotechnol. 10, 313-318 (2015).

28. Maeda, K. \& Mallouk, T. E. Comparison of two- and three-layer restacked Dion-Jacobson phase niobate nanosheets as catalysts for photochemical hydrogen evolution. J. Mater. Chem. 19, 4813-4818 (2009).

29. Yu, S.-H., Lee, S. H., Lee, D. J., Sung, Y.-E. \& Hyeon, T. Conversion reaction-based oxide nanomaterials for lithium ion battery anodes. Small 12, 2146-2172 (2016)

30. Wu, H. B., Chen, J. S., Hng, H. H. \& Wen Lou, X. Nanostructured metal oxide-based materials as advanced anodes for lithium-ion batteries. Nanoscale 4, 2526-2542 (2012)

31. Hu, Y.-Y. et al. Origin of additional capacities in metal oxide lithium-ion battery electrodes. Nat. Mater. 12, 1130-1136 (2013).
32. Poizot, P., Laruelle, S., Grugeon, S., Dupont, L. \& Tarascon, J. M. Nano-sized transition-metal oxides as negative-electrode materials for lithium-ion batteries. Nature 407, 496-499 (2000).

33. Reddy, M. V., Subba Rao, G. V. \& Chowdari, B. V. R. Metal oxides and oxysalts as anode materials for Li ion batteries. Chem. Rev. 113, 5364-5457 (2013).

34. Nitta, N., Wu, F., Lee, J. T. \& Yushin, G. Li-ion battery materials: present and future. Mater. Today 18, 252-264 (2015).

35. Jing, Y., Zhou, Z., Cabrera, C. R. \& Chen, Z. Graphene, inorganic graphene analogs and their composites for lithium ion batteries. J. Mater. Chem. A 2 , 12104-12122 (2014)

36. Zhong, Y., Yang, M., Zhou, X. \& Zhou, Z. Structural design for anodes of lithium-ion batteries: emerging horizons from materials to electrodes. Mater. Horiz. 2, 553-566 (2015).

37. Xu, C., Wang, X. \& Zhu, J. Graphene-metal particle nanocomposites. J. Phys. Chem. C 112, 19841-19845 (2008).

38. Nethravathi, C. \& Rajamathi, M. Chemically modified graphene sheets produced by the solvothermal reduction of colloidal dispersions of graphite oxide. Carbon 46, 1994-1998 (2008).

39. Wang, $\mathrm{H}$. et al. $\mathrm{Mn}_{3} \mathrm{O}_{4}$-graphene hybrid as a high-capacity anode material for lithium ion batteries. J. Am. Chem. Soc. 132, 13978-13980 (2010).

40. Liang, $\mathrm{Y}$. et al. $\mathrm{Co}_{3} \mathrm{O}_{4}$ nanocrystals on graphene as a synergistic catalyst for oxygen reduction reaction. Nat. Mater. 10, 780-786 (2011).

41. Largeot, C. et al. Relation between the ion size and pore size for an electric double-layer capacitor. J. Am. Chem. Soc. 130, 2730-2731 (2008).

42. Ren, Y., Armstrong, A. R., Jiao, F. \& Bruce, P. G. Influence of size on the rate of mesoporous electrodes for lithium batteries. J. Am. Chem. Soc. 132, 996-1004 (2010).

43. Peled, E., Menachem, C., Bar-Tow, D. \& Melman, A. improved graphite anode for lithium-ion batteries chemically: bonded solid electrolyte interface and nanochannel formation. J. Electrochem. Soc. 143, L4-L7 (1996).

44. Laruelle, S. et al. On the origin of the extra electrochemical capacity displayed by MO/Li cells at low potential. J. Electrochem. Soc. 149, A627-A634 (2002).

45. Grugeon, S., Laruelle, S., Dupont, L. \& Tarascon, J. M. An update on the reactivity of nanoparticles Co-based compounds towards Li. Solid State Sci. 5, 895-904 (2003)

46. Fang, Y. et al. Two-dimensional mesoporous carbon nanosheets and their derived graphene nanosheets: synthesis and efficient lithium ion storage. J. Am Chem. Soc. 135, 1524-1530 (2013).

47. Guo, B. et al. A long-life lithium-ion battery with a highly porous $\mathrm{TiNb}_{2} \mathrm{O}_{7}$ anode for large-scale electrical energy storage. Energy Environ. Sci. 7, 2220-2226 (2014).

48. Alcántara, R., Jaraba, M., Lavela, P. \& Tirado, J. L. $\mathrm{NiCo}_{2} \mathrm{O}_{4}$ spinel: first report on a transition metal oxide for the negative electrode of sodium-ion batteries. Chem. Mater. 14, 2847-2848 (2002).

49. Mo, Y. et al. Three-dimensional $\mathrm{NiCo}_{2} \mathrm{O}_{4}$ nanowire arrays: preparation and storage behavior for flexible lithium-ion and sodium-ion batteries with improved electrochemical performance. J. Mater. Chem. A 3, 19765-19773 (2015).

50. Permien, S. et al. Elucidation of the conversion reaction of $\mathrm{CoMnFeO}_{4}$ nanoparticles in lithium ion battery anode via operando studies. ACS Appl. Mater Interfaces 8, 15320-15332 (2016).

51. Permien, S. et al. What happens structurally and electronically during the li conversion reaction of $\mathrm{CoFe}_{2} \mathrm{O}_{4}$ nanoparticles: an operando XAS and XRD investigation. Chem. Mater 28, 434-4444 (2016).

\section{Acknowledgements}

We thank Prof John B. Goodenough and Prof Arumugam Manthiram at the University of Texas at Austin for valuable discussions. G.Y. acknowledges the funding support from the Welch Foundation Grant F-1861, ACS-PRF award (55884-DNI10), and Sloan Research Fellowship. J.L. and K.A. were supported by the U.S. Department of Energy under Contract DE-AC0206CH11357 with the main support provided by the Vehicle Technologies Office, Department of Energy (DOE) Office of Energy Efficiency and Renewable Energy (EERE)

\section{Author contributions}

G.Y. supervised the project; G.Y., L.P. and P.X. conceived the idea; L.P. and P.X. carried out the sample synthesis, structural characterization and electrochemical measurement L.M., Y.Y., Y.Z., D.C., J.L. and K.A. assisted with the in-situ and ex-situ microscopic characterization; L.P., P.X., J.L. and G.Y. co-wrote the paper. All the authors discussed the results, commented on and revised the manuscript.

\section{Additional information}

Supplementary Information accompanies this paper at http://www.nature.com/ naturecommunications

Competing interests: The authors declare no competing financial interests. 
Reprints and permission information is available online at http://npg.nature.com/ reprintsandpermissions/

How to cite this article: Peng, L. et al. Holey two-dimensional transition metal oxide nanosheets for efficient energy storage. Nat. Commun. 8, 15139 doi: 10.1038/ncomms15139 (2017).

Publisher's note: Springer Nature remains neutral with regard to jurisdictional claims in published maps and institutional affiliations. (c) (i) This work is licensed under a Creative Commons Attribution 4.0 International License. The images or other third party material in this article are included in the article's Creative Commons license, unless indicated otherwise in the credit line; if the material is not included under the Creative Commons license, users will need to obtain permission from the license holder to reproduce the material. To view a copy of this license, visit http://creativecommons.org/licenses/by/4.0/

(C) The Author(s) 2017 